\title{
GESTÃO DE RESÍDUOS SÓLIDOS: ESTUDO DE CASO EM UMA INSTITUIÇÃO \\ HOSPITALAR EM BELO HORIZONTE - MG
}

SOLID WASTE MANAGEMENT: A CASE STUDY IN A HOSPITAL INSTITUTION IN

$$
\text { BELO HORIZONTE - MG }
$$

Recebimento: 11/06/2018 - Aceite: 21/12/2018 - Publicação: 31/01/2019

Processo de Avaliação: Double Blind Review

Claudiomiro Werner Chagas ${ }^{1}$

Mestre em Administração

Centro Universitário Unihorizontes

wernerprofessor@yahoo.com.br

Caissa Veloso e Sousa

Doutora em Administração de Empresas

Centro Universitário Unihorizontes

caissaveloso@yahoo.com.br

Gustavo Rodrigues Cunha

Doutor em Administração

Centro Universitário Unihorizontes

gustavo.cunha@unihorizontes.br

Nayara Kelly Batista

Mestranda em Administração

Centro Universitário Unihorizontes

nayarakbatista@yahoo.com.br

\section{RESUMO}

Com os avanços tecnológicos e o aumento da competitividade, emerge a necessidade, tanto legal quanto social, de as empresas descartarem corretamente seus resíduos, atendendo não só às exigências para seu funcionamento, como também as exigências do mercado. Nesse aspecto, a presente pesquisa teve como objetivo principal analisar a gestão de resíduos sólidos segundo a percepção de gestores que atuam em uma organização hospitalar, localizada na região metropolitana de Minas Gerais. O arranjo metodológico se norteou por um estudo de caso de abordagem qualitativa e descritiva, em um hospital localizado no estado de Minas Gerais. A coleta dos dados ocorreu por meio da realização de entrevistas semiestruturadas, a partir de um roteiro com quinze questões, aplicadas a dez gestores da empresa. Os resultados apontaram para a relevância de as organizações terem conhecimento dos resíduos que geram,

${ }^{I}$ Autor para correspondência: R. Alvarenga Peixoto, 1270 - Santo Agostinho, Belo Horizonte - MG, Brasil, 30180-121.

Revista ENIAC Pesquisa, Guarulhos (SP), V.8, n.1, jan.- jun. 2019. 
independentemente de seu grau de periculosidade, tornando-se importante a prática de métodos eficientes com o objetivo de suavizar os impactos negativos gerados pelos resíduos lançados, ao meio ambiente. Assim, a partir de uma segregação eficaz, bem como métodos de cuidado que procuram reduzir o volume de resíduos a serem depositados no solo e relatados à comunidade é um código importante a ser cursado. Observou-se ainda que a reciclagem dos materiais possibilita aumento de receita, bem como diminuição dos custos, pois, quando se recicla um material não se têm os custos com o descarte de tais materiais.

Palavras-chave: Resíduos sólidos; Logística de medicamentos; Gestão ambiental; Logística reversa.

\section{ABSTRACT}

With the technological advances and the increase of the competitiveness emerges the need, both legal and social, of the companies to discard their waste correctly, attending not only to the requirements for its operation, but also the demands of the market. In this aspect, the main objective of this research was to analyze the solid waste management according to the perception of managers who work in a hospital organization, located in the metropolitan region of Minas Gerais. The methodological approach was guided by a qualitative and descriptive case study in a hospital located in the state of Minas Gerais. Data were collected through semi-structured interviews, based on a script with fifteen questions, applied to ten managers of the company. The results pointed to the relevance of the organizations being aware of the residues they generate, regardless of their degree of hazard, and it is important to practice efficient methods with the objective of smoothing the negative impacts generated by the waste released to the environment. Thus, effective segregation as well as methods of care that seek to reduce the volume of waste to be deposited in the soil and reported to the community is an important code to be followed. It was also observed that the recycling of the materials allows an increase of revenue, as well as a reduction of costs, because when a material is recycled one does not have the costs with the disposal of such materials.

Keywords: Solid waste; Logistics of medicines; Environmental management; Reverse logistic.

\section{INTRODUÇÃO}

$\mathrm{Na}$ atualidade, identifica-se uma busca por inovações em produtos, processos e serviços, resultados de ações necessárias para que as organizações possam se manter competitivas no mercado. Tais inovações estão relacionadas às constantes transformações de recursos naturais em produtos e ao ritmo de consumo da sociedade (TADEU, 2012).

Associado ao crescente aumento da população e ao volume cotidiano de consumo de produtos industrializados, esta geração de resíduos transforma-se em uma produção maior de lixo descartável pela sociedade, se comparado ao volume historicamente registrado (CUNHA; CAIXETA FILHO, 2002). Nesse sentido, o processo da coleta e da destinação final dos

Revista ENIAC Pesquisa, Guarulhos (SP), V.8, n.1, jan.- jun. 2019. 
resíduos gerados é apresentado como um dos importantes desafios a serem enfrentados pela sociedade moderna, e sua importância se deve a três fatores: ao grande volume de resíduos gerados, custos financeiros ao seu gerenciamento, e impactos ao meio ambiente e à saúde da população (GONÇALVES; TANAKA; AMEDOMAR, 2013; OLIVEIRA et al., 2017).

Em termos do descarte desse lixo é possível afirmar que mais da metade do lixo produzido no Brasil vai para os aterros sanitários $(58,4 \%)$. O restante se divide entre aterros controlados $(24,2 \%)$, lixões $(17,4 \%)$ compostagem e reciclagem $(7 \%)$ (CONFEDERAÇÃO NACIONAL DA INDÚSTRIA, 2017). Diante da quantidade de resíduos sólidos gerados, torna-se importante que os interesses econômicos se associem aos interesses ambientais, tornando tanto as diretrizes do meio ambiente quanto os resultados financeiros satisfatórios (SANTOS, 2011).

Especificamente na área da saúde, os resíduos gerados pelo setor devem receber tratamento especial ao serem descartados, dado que seu descarte incorreto pode contaminar a água e o solo e trazer malefícios à saúde humana e animal (SOUZA et al., 2013; LOPES; CALABRESE, 2016). Assim, a gestão de resíduos sólidos se apresenta como uma estratégia importante, não apenas sob a ótica organizacional, mas, sobretudo, socioambiental.

A partir do contexto apresentado, este estudo objetivou apresentar a análise da gestão de resíduos sólidos, segundo a percepção de gestores que atuam em uma organização hospitalar, localizada na Região Metropolitana de Belo Horizonte, Minas Gerais. A seguir, apresenta-se a fundamentação teórica do estudo.

\section{SUSTENTABILIDADE}

A sustentabilidade deve englobar o conceito de responsabilidade social relacionado à população, bem como às organizações que mantêm ligação direta com o tema. As organizações são compreendidas como os principais agentes econômicos, visto que propiciam o desenvolvimento de muitos países por meio da exploração de reservas naturais, emitido gases estufas, aumentando a poluição global. Em contrapartida, estas mesmas empresas podem contribuir para a sustentabilidade, inovando e renovando o processo de produção, por intermédio das avançadas tecnologias disponíveis atualmente (RICO, 2010; FREITAS; FREITAS, 2016).

Revista ENIAC Pesquisa, Guarulhos (SP), V.8, n.1, jan.- jun. 2019. 
Savitz e Weber (2007) evidenciam que as empresas têm papel fundamental como transformadoras da sociedade, às quais são atribuídas as responsabilidades de promoverem ações que melhorem a dignidade humana, sem praticarem ações que possam ser prejudiciais a seu público, consumidores, fornecedores e à sociedade que já está consolidada. Para efetuar estas ações é preciso que a empresa resguarde seu capital financeiro e seus patrimônios, a fim de garantir o desenvolvimento do negócio e manter suas obrigações monetárias. Ainda, na prática, a sustentabilidade engloba gestão, estratégia e lucro, pois são as esferas sociais, econômicas e ambientais que acarretam perigos e chances de crescimento que impactam as empresas e os negócios de modo geral.

\section{GESTÃO DE RESÍDUOS SÓLIDOS}

O Conama, na resolução N 05/93, apresenta o conceito de resíduos sólidos, baseado pela NBR 10.004/87 da ABNT, como sendo aqueles resíduos sólidos e semissólidos resultantes das atividades industriais, domésticas, hospitalar, comercial, agrícola, de serviços e de varrição.

A gestão de resíduos sólidos é uma questão que envolve todo o processo produtivo de uma organização, desde a matéria-prima, passando pelos métodos e processos de produção, até a distribuição ao cliente final (SCHOOT FILHO, 2017), assim, gerenciar os resíduos, de forma integrada, significa cuidar do "berço" ao "túmulo" (ALMEIDA, 2012).

Interessante sublinhar que, em função do crescimento acelerado do descarte inadequado de resíduos, notam-se alterações expressivas em sua composição e características, bem como no aumento de periculosidade (EPA, 2008; OMS, 2013). Tal contexto, associado ao aumento progressivo do custo para eliminação de resíduos sólidos, pode ser visto como um incentivo para adequar as boas práticas de gestão destes resíduos (MASSAWE, 2014). Para tal, deve-se considerar que o gerenciamento destes tipos de resíduos possui responsabilidades determinadas em legislações sobre os resíduos e acarretam em sistemas de coleta, tratamento, transporte e disposição final (JACOBI; BESEN, 2006).

Os tipos de resíduos sólidos que chamam a atenção de gestores, órgãos públicos e sociedade, e que merecem destaque, não só pelas quantidades geradas, mas, principalmente, pelas consequências danosas causadas pelo descarte incorreto, são os Resíduos de Serviços de

Revista ENIAC Pesquisa, Guarulhos (SP), V.8, n.1, jan.- jun. 2019. 
Saúde (RSS) (RIBEIRO; PEREIRA, 2015; LOPES; CALABRESE, 2016). O conceito de RSS, de maneira abrangente, pode ser entendido como resíduos líquidos ou semissólidos que são gerados dentro de estabelecimentos de saúde (RISSO, 1993; CASTRO et al., 2017).

A gestão dos resíduos sólidos pode promover a redução das quantidades de resíduos a serem descartados, reciclados ou incinerados, e ainda aumentar o reaproveitamento de materiais. Portanto, a segregação eficiente dos RSS evita a contaminação dos demais resíduos, reduz riscos à saúde, permitindo a recuperação dos materiais recicláveis, assim como a redução dos custos em coletar, transportar e tratar os RSS (BARBOSA et al., 2017).

Portanto, a gestão e o gerenciamento de Resíduos de Serviços de Saúde abordam condições de implementação e acompanhamento constantes, exigindo dos gestores providências também constantes, facilitando a tomada de decisões e abrangendo as atividades necessárias para o cumprimento das legislações vigentes, de forma mais eficiente e eficaz (MARANHÃO, 2012; FERREIRA, 2017).

\section{METODOLOGIA}

A presente pesquisa caracteriza-se como um estudo de caso descritivo de abordagem qualitativa. A unidade de análise compreendeu uma empresa do setor de saúde, situada na cidade de Belo Horizonte/MG, denominada com o nome fictício de Hospital Alfa.

O Hospital Alfa, assim denominado neste estudo, foi instalado no final do século XIX, mais precisamente em 1899, como sendo a primeira instituição de saúde de Belo Horizonte/MG. Hoje está localizada na região conhecida como região hospitalar, no bairro de Santa Efigênia.

Para a composição da pesquisa foram entrevistados dez gestores responsáveis pelo processo de gestão ambiental da organização e pelo processo de armazenagem. A escolha da organização deveu-se à acessibilidade dos sujeitos de pesquisa por constituírem atores diretamente envolvidos com o processo de interesse desta.

$\mathrm{Na}$ coleta dos dados utilizaram-se entrevistas e conversas relacionadas ao tema proposto na pesquisa, por meio de roteiro semiestruturado previamente construído, com quinze questões relacionadas aos objetivos da pesquisa. O roteiro contemplou duas categorias

Revista ENIAC Pesquisa, Guarulhos (SP), V.8, n.1, jan.- jun. 2019. 
de análise: meio ambiente e resíduos no hospital, que foram estabelecidas a partir do objetivo traçado.

Com base nos dados obtidos por meio da entrevista semiestruturada utilizaram-se as respostas dos entrevistados para se verificar a percepção dos funcionários sobre os resultados da gestão de resíduos sólidos dentro da organização, o reaproveitamento e o descarte dos resíduos gerados no processo. Para tanto, a análise de dados ocorreu por meio da análise de conteúdo que, para Bardin (2008), prevê as seguintes etapas: pré-análise; exploração do material e tratamento dos resultados; inferência e interpretação.

\section{APRESENTAÇÃO E ANÁLISE DOS RESULTADOS}

No que tange ao perfil dos sujeitos, a maioria deles é do sexo masculino, com idades variando entre 22 e 57 anos; a maioria não possui filhos e, quanto ao tempo de trabalho na instituição, a média é de 5 a 9 anos; em relação ao tempo de trabalho no cargo, a média é de 5 e 6 anos. Para preservar as identidades dos sujeitos de pesquisa, estes foram caracterizados com a letra $\mathrm{E}$, seguida da disposição numérica correspondente à realização em sequência das entrevistas.

A análise de cada uma das categorias é exposta a seguir de maneira detalhada, para que haja a compreensão dos resultados obtidos com das entrevistas.

\subsection{PROCESSO DE CLASSIFICAÇÃO DOS MEDICAMENTOS}

Ao analisar os depoimentos dos gestores entrevistados pode-se identificar, no que tange ao processo de classificação dos medicamentos, que tal processo acontece de acordo com a legislação vigente. No caso estudado, pauta-se na resolução 306 que dispõe sobre o Regulamento Técnico para o gerenciamento de resíduos de serviços de saúde, bem como na resolução CONAMA 358 que dispõe sobre o tratamento e a disposição final dos resíduos dos serviços de saúde e dá outras providências.

Bom, em um estabelecimento hospitalar existe uma legislação que é a RDC 306 e a CONAMA 358. Nela cita o descarte de resíduos de uma forma geral, onde que tá contemplado os medicamentos. Então é classificado como, são cinco grupos, o grupo A B C D e E. e o grupo B que são os resíduos químicos, tal, existe os

Revista ENIAC Pesquisa, Guarulhos (SP), V.8, n.1, jan.- jun. 2019. 
medicamentos, tá classificado os medicamentos, são os medicamentos vencidos que devem ser encaminhados para tratamento (E5).

Em consonância aos relatos dos entrevistados, Andreoli, Dias e Konan, (2014) destacam o alto impacto dos medicamentos na gestão logística, tendo em vista que são materiais com expressiva importância para o processo de atenção e cuidados ao paciente, além de serem determinantes para os gastos de uma unidade de saúde.

Os entrevistados declararam existir controle extenso do mapeamento dos medicamentos vencidos. No entanto, é importante salientar que antes de determinar soluções de tratamento ou destinação final dos resíduos já gerados, é preciso pensar em alternativas do ponto de vista ambiental, com vista a redução desses resíduos, buscando técnicas de reuso somente quando não existir a possibilidade de utilização de tais alternativas. Neste contexto, cabe salientar que o sistema informatizado, utilizado pela instituição, não possibilita o controle adequado desses resíduos.

\subsection{DESTINAÇÃO DOS MEDICAMENTOS VENCIDOS}

Com relação à forma de destinação de medicamentos vencidos ou pouco consumidos, identificou-se que estes são encaminhados para incineração, por meio de um contrato estabelecido com uma empresa terceirizada. Após esse processo, o medicamento é conduzido para um aterro destinado a resíduos perigosos, como evidenciado no relato a seguir:

Bom, a destinação deles igual eu falei, é a incineração, medicamento vencido ele tem que ser incinerado, então tem-se contrato celebrado com essa com essa empresa e depois ela é encaminhada para um aterro né, É Um aterro controlado só para resíduos incinerados, então é a cinza encaminhado para este tipo de aterro classe 1 (E1).

Portanto, no que tange à destinação adequada de tais medicamentos, cabe reportar às colocações de Leite e Brito (2000), na qual os autores ressaltam que se faz necessário utilizar técnicas de separação adequadas, além do tratamento e destinação final de forma que não haja prejuízos ao meio ambiente ecologicamente equilibrado.

Revista ENIAC Pesquisa, Guarulhos (SP), V.8, n.1, jan.- jun. 2019. 


\subsection{PROCESSO DE LOGÍSTICA REVERSA}

De forma geral, a farmácia efetua a devolução do medicamento para o setor de almoxarifado que solicita à contabilidade a nota fiscal da compra e, em seguida, é realizado o contato com o fornecedor para realizar a devolução do medicamento. No entanto, a partir do discurso dos entrevistados, observou-se dificuldades dos grandes hospitais nesse processo. No ano de 2010, a logística reversa, em se tratando de questões ambientais, foi contemplada pela política nacional de resíduos sólidos, entretanto, na prática a realidade é outra. Por diversas vezes, o contato com o fabricante é conflituoso, devido a inabilidade em recolher esse medicamento e tratar o resíduo de forma ambientalmente correta, ficando por responsabilidade da instituição a destinação correta desses medicamentos: "A farmácia devolve para o almoxarifado, o almoxarifado solicita na contabilidade a nota de devolução e com a nota de devolução a gente entra em contato com fornecedor para recolher (E4)."

Tais achados corroboram a visão de Bartholomeu e Caixeta-Filho (2011), que afirmam que, no Brasil, atualmente, é constatado o aumento nos índices de poluição e grande deterioração ambiental nos lugares em que a gestão ambiental não trabalha com excelência, visto que há baixo controle e pouca fiscalização dos órgãos responsáveis.

Os entrevistados também demonstraram cuidado por parte da instituição, no que tange à política ambiental, tendo em vista que a destinação desses medicamentos precisa ocorrer da maneira adequada, pois se trata de materiais de alto nível de contaminação.

\subsection{DESCARTE DE MEDICAMENTOS}

Em relação aos canais utilizados para o descarte de medicamentos, os entrevistados relataram que o mesmo acontece por meio de bombonas, reservatórios utilizados para o condicionamento de medicamentos vencidos. As bombonas são colocadas nas áreas em que se utilizam mais produtos químicos.

Nesse sentido, identifica-se que os resíduos gerados pelo setor da saúde devem receber um tratamento especial quando descartados, tendo em vista que, quando ocorre o descarte de forma incorreta, esses resíduos contaminam a água e o solo. E mesmo o Estado tendo implementado políticas públicas que protejam o meio ambiente, faz-se necessário que as

Revista ENIAC Pesquisa, Guarulhos (SP), V.8, n.1, jan.- jun. 2019. 
empresas adotem políticas de responsabilidade socioambiental, e o consumidor se conscientize e contribua para o descarte de maneira correta (SHIBAO et al., 2010).

Desse modo, esta pesquisa buscou identificar se o descarte utilizado pela Instituição é ambientalmente sustentável. De forma unânime, os entrevistados verbalizaram cuidado por parte da Instituição em atender às exigências legais e éticas com o descarte de todos os tipos de resíduos. A Instituição encaminha para reciclagem todos os resíduos possíveis, com vistas a garantir que esse resíduo volte a seu ciclo inicial. Com relação aos medicamentos, sejam vencidos ou inapropriados para o uso, são estes destinados à finalidade adequada.

Segundo Leite e Brito (2000), as áreas de atuação da logística reversa possuem vantagens econômicas por meio de sua utilização no contexto das organizações. Os benefícios estão associados aos objetivos econômicos da implantação relacionados à redução de custos, devido ao aproveitamento de matéria-prima derivadas do processo de reciclagem, assim como a revalorização dos bens pelo reprocesso e reutilização.

Assim, buscou-se analisar se as ações ou Logística Reversa e Reciclagem, adotadas pela empresa, contribuem para a geração de receita, segundo a percepção dos entrevistados. De forma unânime, os entrevistados verbalizaram positivamente, o que possibilitou inferir que os materiais reciclados podem ser comercializados a fim de trazer recursos financeiros à Instituição, e, por conseguinte aumentando a receita, e diminuindo os custos, pois, quando se recicla um material, os custos são menores, como pôde ser observado no relato de E8.

Sim, no caso da reciclagem a gente vende papelão, papel, plástico, óleo, esses materiais todos são usados pra reciclagem e a gente tem uma, a gente recebe dinheiro sobre esses resíduos, pelo peso deles, a empresa tem esse ganho (E8).

A esse respeito, Liva, Pontelo e Oliveira (2005) ressaltam que, com o aumento da reciclagem e reutilização dos produtos, e a diminuição da geração de resíduos, diminuem-se os custos, porque retornam-se as matérias a seus ciclos produtivos, melhorando a imagem da empresa perante o mercado, pois se torna uma empresa ambientalmente responsável, o que traz uma imagem publicitária forte e positiva.

Questionou-se aos entrevistados se eles consideram que algo poderia ser feito de forma diferente. Eles concordam que a instituição se empenha em atender à legislação e se preocupa em estabelecer estratégias que visem ao cuidado com o meio ambiente.

Revista ENIAC Pesquisa, Guarulhos (SP), V.8, n.1, jan.- jun. 2019. 
Nesse aspecto, Arima e Battaglia (2007) argumentam que é importante que exista mercado para produtos reciclados, pois, caso contrário, as empresas que utilizam o material reciclado como matéria-prima irão diminuir a produção de materiais, diminuindo assim a demanda por matéria-prima reciclada.

\section{CONSIDERAÇÕES FINAIS}

O presente estudo desenvolveu-se com o objetivo de analisar a gestão de resíduos sólidos em uma organização hospitalar, localizada na Região Metropolitana de Belo Horizonte, Minas Gerais, segundo a percepção de gestores responsáveis pelos processos relacionados. Para tal, metodologicamente, desenvolveu-se um estudo de caso descritivo, de abordagem qualitativa, realizado em uma organização do segmento hospitalar.

Dentre os principais resultados deste estudo pode-se destacar, em primeira instância, o processo de mapeamento e o descarte dos resíduos hospitalares. Nota-se que, na organização em questão, são feitos esforços para se adquirirem somente produtos com a data de validade superior a seis meses da data da prescrita na nota de compra, para, assim, reduzir o descarte de materiais. Importante ressaltar que os medicamentos vencidos e/ou pouco consumidos são encaminhados para o processo de incineração e, posteriormente, destinados aos aterros correspondentes.

Nesse mesmo sentido, outro ponto relevante a ser destacado refere-se ao descarte de medicamentos já vencidos, questão de considerável relevância, dados os impactos negativos que podem gerar tanto para a saúde humana quanto para o restante do meio ambiente. Observa-se, pois, que os medicamentos nessas condições, na organização, são coletados, transportados e incinerados. Este processo ocorre por empresas terceirizadas e credenciadas em órgãos ambientais. Isso é considerado um avanço para o hospital, devido a estes medicamentos serem encaminhados para o local de destino correto. Nesse aspecto destaca-se que a organização estudada compreende um hospital de grande porte e, por conseguinte, suas ações são vistas como uma "vitrine", o que a deixaria exposta caso não procedesse o correto descarte desses produtos.

Ressalta-se, ainda, a relevância das organizações em ter conhecimento dos resíduos que geram, independente do seu grau de periculosidade, tornando-se importante a prática de 
métodos eficientes que tenham, por objetivo, suavizar os impactos negativos gerados pelos resíduos lançados ao meio ambiente.

Nesta análise ainda se verificou que os profissionais que coordenam essa questão estão procurando continuamente informações em relação ao descarte correto dos resíduos para promoverem a adequação do hospital às exigências das normas e legislações relacionadas aos RSS.

Destacada como um fator importante na continuidade deste plano de gerenciamento de resíduos é a persistência dos profissionais, uma vez que as mudanças e adaptações acontecem tão somente a médio e longo prazo. Vale destacar, portanto, que atualmente o plano de gerenciamento está em funcionamento, ressaltando que o Hospital Alfa foi um pioneiro na cidade de Belo Horizonte adequando-se à legislação vigente.

Dentre de alguns problemas encontrados pelos gestores para atenderem essas normas, a falta de espaço físico é um grande desafio, porque a estrutura do hospital é antiga e não visava, nos primórdios, tais ações de gestão. Esta ausência de espaço físico adequado dificulta a circulação dos carrinhos coletores dos resíduos dentro do estabelecimento, principalmente porque o corredor utilizado para manusear estes RSS é o mesmo utilizado por visitantes, funcionários e pacientes da instituição.

Dentre os principais pontos críticos salientados pelos entrevistados deste estudo, identifica-se a adoção de incineração para os resíduos de saúde que, além de gerar um dispêndio desnecessário de recursos financeiros e de representar uma agressão ao meio ambiente, esse processo causa transtornos às comunidades próximas aos incineradores que sofrem com o lançamento de componentes tóxicos no ar, além da exposição ao risco no transporte do gerador ao incinerador.

Tais achados permitem concluir a importância da evolução na legislação, no que tange à gestão de resíduos sólidos, bem como maior conscientização da sociedade que tornará mais fortalecida a questão ambiental. Visando à lucratividade nas etapas de coleta e reciclagem, procurando implantar os materiais reciclados novamente no mercado comercial.

Em relação às limitações encontradas para a realização desta pesquisa, destaca-se, principalmente, a dificuldade de disponibilidade dos entrevistados em conceder as entrevistas devido à agenda de trabalho. Ressalta-se, ainda, o conhecimento restrito por parte deles, bem

Revista ENIAC Pesquisa, Guarulhos (SP), V.8, n.1, jan.- jun. 2019. 
como a necessidade de se estabelecer estratégias em conseguir informações importantes, porém limitadas à instituição.

É importante ressaltar que os resultados disponibilizados pelo estudo não possuem a intenção de serem generalizados para todas as instituições hospitalares, tendo em vista que cada instituição possui suas particularidades. Portanto, sugere-se, para estudos futuros, pesquisas em outras instituições hospitalares, com vistas a possíveis comparações e ampliação dos achados.

\section{REFERÊNCIAS}

ALMEIDA, M. V. A distribuição física como recurso estratégico na obtenção de vantagem competitiva no segmento de bens de consumo de massa no Brasil. 2012. $125 \mathrm{f}$. Dissertação (Mestrado em Administração) - Universidade Presbiteriana Mackenzie, São Paulo. 2012.

ANDREOLI, G.L.M.; DIAS, C.N.; KONAN, A.T.B. Planejamento e gestão logística de medicamentos em uma Central de abastecimento farmacêutico hospitalar. SIMPOI, 2014.

ARIMA, S.; BATTAGLIA, A. Da terra para a terra, uma visão do ciclo total. Tecnologística, São Paulo: Publicare, 2007.

BARBOSA, S. et al. Gestão de Resíduos de Saúde: estudo de caso-Pelotas, gerenciamento no ano de 2006 e legislação pertinente. In: Forum Internacional de Resíduos Sólidos-Anais. 2017.

BARDIN, Laurence. Análise de conteúdo. 19. ed. Lisboa: Edições 70, 2008.

BARTHOLOMEU, D. B.; CAIXETA-FILHO, J. V. Logística Ambiental de Resíduos Sólidos. São Paulo: Atlas, 2011.

BRASIL. Lei n. 12. 305, de 2 de agosto de 2010. Política Nacional de Resíduos Sólidos. 2. ed. Brasília: Câmara dos Deputados, Edições Câmara, 73 p., 2012.

CASTRO, J. M. et al. Implicações dos Resíduos Sólidos a Saúde Humana: Explorando Publicações de Enfermagem. UNICIÊNCIAS, v. 21, n. 1, p. 45-49, 2017.

CONFEDERAÇÃO NACIONAL DA INDÚSTRIA. Sustentabilidade na indústria da alimentação: uma visão de futuro para a Rio+20. Associação Brasileira das Indústrias da Alimentação. Brasília: CNI, 2012.

Revista ENIAC Pesquisa, Guarulhos (SP), V.8, n.1, jan.- jun. 2019. 
CUNHA, V.; CAIXETA FILHO, J. V. Gerenciamento da coleta de resíduos sólidos urbanos: estruturação e aplicação de modelo não-linear de programação por metas. Gestão e Produção, v.9, n.2, p.143-161, Ago. 2002.

EPA -Environment Protection Agency. Climate Change and Waste. Reducing Waste Can Make a 2008. Difference. Disponível em: < http://nepis.epa.gov/Exe/ZyPDF.cgi/9101NS45.PDF?Dockey=9101NS45.PDF. Acesso em: 15.fev 2018

FERREIRA, E. R. Gestão e Gerenciamento dos Resíduos de Serviços de Saúde em Presidente Prudente - SP. Dissertação de Mestrado. Programa de Pós-graduação em Geografia, UNESP. Presidente Prudente - SP, 2017.

FREITAS, M.; FREITAS, M. C. S. A sustentabilidade como paradigma: cultura, ciência e cidadania. Petrópolis-RJ: Vozes, 2016.

GONÇALVES, M. A; TANAKA, A. K; AMEDOMAR, A. A. A destinação final dos resíduos sólidos urbanos: alternativas para a cidade de São Paulo através de casos de sucesso.

Future Studies Research Journal, São Paulo, v. 5, n. 1, pp. 96-129, jan./jun. 2013.

JACOBI, P. R.; BESEN, G. R. Gestão de resíduos sólidos em São Paulo: desafios da sustentabilidade. Estudos Avançados, v. 25, n.71, 2011.

LEITE, P. R.; BRITO, E. P. Z. Fatores que influenciam a reciclagem de materiais em canais de distribuição reversos [CD-ROM]. In: Anais... Encontro da Associação Nacional de PósGraduação e Pesquisa em Administração, 24. Florianópolis, 2000.

LIVA, P. B. G.; PONTELO, V. S L.; OLIVEIRA, W. S. Logística Reversa I, 2005. Disponível em : <http:www.techoje.com. br/site/techoje/categoria/abrirPDF/301>. Acesso em: 18 jan. 2018.

LOPES, D. A. C.; CALABRESE, I. Gerenciamento De Resíduos De Serviço De Saúde. Revista Saúde, v. 10, 2016.

MASSAWE, C. Sustentabilidade nas organizações: a aplicação do método Gaia de gerenciamento de impactos ambientais em uma empresa. Revista de Gestão Ambiental e Sustentabilidade - GeAS, 4(3), 103-121, 2014.

MARANHÃO, R. A. Gestão de Resíduos de Serviço de Saúde em Organização Militar da Marinha do Brasil: um estudo na Base Naval do Rio de Janeiro. Monografia de MBA em Gestão e Tecnologias Ambientais, Escola Politécnica da Universidade de São Paulo, 2012.

OLIVEIRA, K. N. et al. Simulação em processo de triagem de materiais recicláveis. Revista Eniac Pesquisa, v. 6, n. 1, p. 93-108, 2017.

ORGANIZAÇÃO MUNDIAL DA SAÚDE (OMS). World health report 2013: Research for universal health coverage. Disponível em: $<$ http://www.who.int/whr/en/> . Acesso em: 13 fev. 2018

Revista ENIAC Pesquisa, Guarulhos (SP), V.8, n.1, jan.- jun. 2019. 
RICO, E. M. RESPONSABILIDADE SOCIAL EMPRESARIAL E SUSTENTABILIDADE. Management in Dialogue Review, v. 12, n. 1, 2010.

RIBEIRO, L. M. P.; PEREIRA, J. R. Relações entre poder público e sociedade na gestão de resíduos sólidos de um município de minas gerais: uma análise sob o enfoque das abordagens da administração pública. Management in Dialogue Review, v. 16, n. 2, 2015.

RISSO, W.M. Gerenciamento de resíduos de serviços de saúde: a caracterização como instrumento básico para abordagem do problema. 1993.162 f. Dissertação (Mestrado) em Saúde Pública - Faculdade de Saúde Pública da Universidade de São Paulo - Departamento de Saúde Ambiental. São Paulo,1993.

SANTOS, A. Projeto para implantação do plano de gerenciamento de resíduos de serviços de saúde em uma unidade mista do município de Custódia-PE. 2011. 29f. Monografia (Trabalho de Conclusão de Curso) - Fundação Oswaldo Cruz: Recife, 2011.

SAVITZ, A. W.; WEBER, K. A empresa sustentável: o verdadeiro sucesso é o lucro com responsabilidade social e ambiental. Rio de Janeiro: Elsevier, 2007.

SHIBAO, F. Y.; MOORI, R. G.; SANTOS, M. R. A logística reversa e a sustentabilidade empresarial. In: Seminários em Administração- SemeAd, 2010,.13, São Paulo/SP. Anais... São Paulo, SemeAd, 2010.

SCHOOT - FILHO, O. Panorama de coleta seletiva no Brasil: Desafios e perspectivas a partir de três estudos de casos. INTERFACEHS - Revista de Gestão Integrada em Saúde do Trabalho e Meio Ambiente. V.2. n.4, Ago 2017.

SOUZA, F. P., et al. Viabilidade da aplicação da logística reversa no gerenciamento dos resíduos dos serviços de saúde: um estudo de caso no hospital X. Perspectiva online: Ciências exatas e engenharia. Campos dos Goytacazes, v. 3, n. 6, p. 56-72, 2013.

TADEU, H. F. B. et al. Logística Reversa e Sustentabilidade. São Paulo: CENGAGE LEARNING, 2012.

Revista ENIAC Pesquisa, Guarulhos (SP), V.8, n.1, jan.- jun. 2019. 